\title{
Modelo de conductancia hidráulica de la dentina humana ex vivo
}

\section{Hydraulic conductance model of ex vivo human dentin}

\author{
Hevia $\mathrm{J}^{1}$, Fresno $\mathrm{C}^{2}$, Martín $\mathrm{J}^{2}$, Moncada $\mathrm{G}^{3}$, Letelier $\mathrm{C}^{4}$, Oliveira Junior OB ${ }^{5}$, Fernández $\mathrm{E}^{6}$
}

\section{RESUMEN}

El objetivo principal de este trabajo fue montar y probar un modelo experimental para medir la conductancia hidráulica de la dentina ex vivo. Diecisiete terceros molares sanos, con indicación de exodoncia, de donantes sanos de edades entre 15 y 30 años fueron obtenidos mediante consentimiento informado. Luego de limpiarlos, desinfectarlos, incluirlos en resina epóxica y cortarlos se obtuvieron 17 muestras de dentina, correspondiente a un disco de resina con un corte coronal de diente que presenta dentina expuesta en ambas caras de éste. Tres equipos para medir la conductancia hidráulica de la dentina fueron armados siguiendo la descripción del modelo de Pashley. Las muestras fueron instaladas en una cámara de difusión, conectada mediante tubos de silicona a una pipeta graduada y una columna de agua de $20 \mathrm{~cm}$. Mediante el desplazamiento de una burbuja de agua al interior de la pipeta, se midió la conductancia hidráulica de cada muestra, 3 veces los días 14, 21, 28 y 35 postextracción. Los datos fueron tabulados y analizados estadísticamente. No existe diferencia en la tasa de flujo de una muestra medida en los tres equipos ( $p=0.5937$ ). No existe diferencia en las mediciones de la conductancia hidráulica de 13 muestras de dentina humana medida en los días $14,21,28$ y 35 postextracción $(p=0.0704)$. Es posible montar un modelo experimental para estudiar la conductancia hidráulica de la dentina ex vivo, basado en el modelo de Pashley y col. El modelo pareciera ser confiable, pero es necesaria más investigación para poder validar su confiabilidad.

Rev. Clin. Periodoncia Implantol. Rehabil. Oral Vol. 6(3); 114-117, 2013.

Palabras clave: Dentina, conductancia, difusión.

\section{ABSTRACT}

The main objective of this work was to mount and test an experimental model to measure the hydraulic conductance of ex vivo dentin. Seventeen healthy third molars, with indication of extraction of healthy donors aged between 15 and 30 years were obtained by informed consent. After cleaning them, disinfecting them, including them in resin epoxy and cutting them, there were 17 samples of dentin, corresponding to a disk of resin with a coronal section of tooth showing the dentin exposed on both sides of it. Three machines to measure the hydraulic conductance of the dentin were assembled according to the description of the model of Pashley. Samples were installed in a Chamber of diffusion, connected by using silicone tubes to a graduated transfer pipette and a $20 \mathrm{~cm}$ water column. Through the displacement of a bubble of water in the inside of the pipette, the hydraulic conductance of each sample was measured 3 times on the $14^{\text {th }}, 21^{\text {st }}, 28^{\text {th }}$ and $35^{\text {th }}$ day post extraction. The data were tabulated and analyzed statistically. There is no SS difference in the rate of flow of a measured sample in the three machines ( $p=0.5937$ ). There is no SS difference in measurements of the hydraulic conductance of 13 samples of human dentin measured in days $14,21,28$ and 35 postextraction $(p=0.0704)$. It is possible to mount an experimental model to study the hydraulic conductance of dentin ex vivo, based on the model of Pashley. The model seems to be reliable, but more research is needed in order to validate its reliability.

Rev. Clin. Periodoncia Implantol. Rehabil. Oral Vol. 6(3); 114-117, 2013.

Key words: Dentin, conductance, diffusion.

\section{INTRODUCCIÓN}

La dentina forma la estructura central de los dientes. Su espesor está atravesado por túbulos que se extienden desde la pulpa hasta el límite amelodentinario. Estos túbulos le confieren la característica física de permeabilidad ${ }^{(1)}$.

La permeabilidad de un material puede ser definida como la capacidad para permitir el paso de un solvente o solución a través de él. Para distinguir esta diferencia, la conductancia hidráulica es definida como la capacidad de un material para permitir el paso de agua destilada a través de él(2).

La primera descripción de permeabilidad de sustancias a través de la dentina fue dada por Fritsch en 1914. En la década de los 40, Lefkowits observó como un colorante inyectado en la pulpa de un diente, penetró en toda la dentina en un poco más de media hora. Algunos años después, Bodecker y Lefkowitz observaron como el colorante de un material de obturación puesto en una cavidad profunda, fue capaz de pasar a zonas adyacentes del esmalte, dentina y pulpa. A partir de estos estudios se comienza a definir el concepto de "permeabilidad dentinaria", que en la actualidad se define como el pasaje de fluidos, iones, moléculas, partículas y bacterias en y a través de la dentina bajo diferentes condiciones ${ }^{(3)}$.

Pashley y cols. han estudiado la relación de la permeabilidad de la dentina con su topografía, morfología y con distintas soluciones ${ }^{(4,5)}$ También se ha investigado la permeabilidad de diversos componentes de los materiales dentales y su posible efecto citotóxico en las células pulpares $^{(2,6-9)}$. Otra línea que se ha desarrollado en los estudios de permeabilidad dentinaria, es la prueba de biocompatibilidad de sistemas adhesivos $^{(6)}$. Producto de la hidratación y naturaleza compleja de éste tejido, la adhesión a la dentina y el sellado de las superficies dentinarias expuestas continúa siendo una problemática a pesar de los avances en la odontología adhesiva. Modelos de permeabilidad han sido usados para

1. Cirujano Dentista. Universidad de Chile. Chile.

2. Profesora Asistente. Departamento Odontología Restauradora, Universidad de Chile. Chile.

3. Profesor Titular. Departamento Odontología Restauradora, Universidad de Chile. Chile.

4. Instructor. Departamento Odontología Restauradora, Universidad de Chile. Chile.

5. Profesor. Departamento de Odontologia Restauradora, Disciplina de Dentística. Univ. Estadual Paulista, UNESP. School of Dentistry of Araraquara. Brasil. 6. Profesor Asistente. Director de Departamento Odontología Restauradora, Universidad de Chile. Chile. Programa PHD en Ciencias Odontológicas Mención Restauradora. Univ. Estadual Paulista, UNESP. Brasil. 
calcular la capacidad de los sistemas adhesivos para sellar la dentina y para determinar la fuerza de adhesión a ella de distintos sistemas adhesivos, cementos y otros materiales dentales.

El presente trabajo, tomará la descripción de un modelo experimental que se ha usado desde $1974^{(5)}$. Será montado utilizando materiales comunes y fáciles de obtener, y puesto en marcha para establecer la confiabilidad de las mediciones de conductancia hidráulica (permeabilidad de agua destilada) de dentina humana ex vivo.

\section{MATERIALES Y MÉTODOS}

El presente estudio correspondió a un estudio observacional ex vivo. Este estudio fue aprobado por el Comité de Ética de la Facultad de Odontología de la Universidad de Chile (PRI ODO 0304/2012).

\section{Muestra}

La muestra correspondió a 17 terceros molares humanos superiores e inferiores.Los criterios de inclusión utilizados fueron: dientes sanos, libres de caries con indicación de exodoncia, de pacientes adultos sanos ASA I de edades entre 15 y 30 años, que fueron extraídos en una sola pieza sin la necesidad de realizar odontosección. Todos los participantes del estudio donaron sus terceros previa firma de un consentimiento informado. En el caso de menores de edad, dicho consentimiento fue firmado por su representante legal.

Inmediatamente después de la exodoncia, cada especimen fue limpiado con gasa y suero fisiológico e identificado para almacenar en forma individual durante todo el transcurso del estudio. Cada uno fue desinfectado sumergiéndolo durante 24 horas en una solución de timol al $0.1 \%$. Posteriormente, cada molar fue enjuagado y mantenido inmerso en solución de Hank HBSS (Sigma ${ }^{\circledR}$ Hanks' Balanced salt solution) ${ }^{(10)}$.

Entre el día 2 y 4 postextracción, cada molar fue sacado del medio de conservación para ser incluido en un bloque cilíndrico de resina epóxica de $25 \mathrm{~mm}$ de diámetro. Para esto cada molar fue limpiado para eliminar todos los restos de ligamento periodontal usando curetas (HuFriedy® 5-6). Luego, y para asegurar un sellado hermético e íntimo contacto entre la superficie de la resina epóxica y del molar, el esmalte coronal de cada molar fue sometido a tratamiento de superficie con ácido fosfórico al $35 \%$ (Coltène ${ }^{\circledR}$ Etchant Gel S) por 30 segundos, lavado con agua por 60 segundos, secado con aire y pincelado con cianoacrilato (Ceys $®$ SuperCeys Pincel).

Los molares fueron situados en el interior de un molde cilíndrico de $25 \mathrm{~mm}$ de diámetro, apoyados sobre la cara oclusal en el centro del molde haciendo coincidir su eje mayor con el eje del cilindro. Cada molde fue vaciado con resina epóxica, esperando la polimerización durante 48 horas. Posteriormente, los bloques de resina epóxica fueron sacados de sus moldes y se obtuvieron cilindros de $25 \mathrm{~mm}$ de diámetro.

Con el objetivo de obtener discos de dentina de $1 \mathrm{~mm}$ de grosor, se realizaron 2 cortes perpendiculares al eje mayor usando la recortadora Isomet 1000 (BuehlerLerLakebuff $囚$ ). Un primer corte coronal para eliminar las cúspides y exponer la dentina y un segundo corte $1 \mathrm{~mm}$ hacia cervical. Los cortes se realizaron a 700rpm y $500 \mathrm{gr}$ de presión bajo abundante refrigeración por agua. Una vez realizado el primer corte todos los cilindros fueron observados con lupa, para asegurarse que el esmalte oclusal hubiese sido completamente eliminado. La superficie fue lijada con papel abrasivo (n. 600 Siliconcarbide) bajo agua circulando para regularizarla. Luego de ambos cortes se obtuvo un disco cilíndrico de $1 \mathrm{~mm}(+/-0.1 \mathrm{~mm})$ de grosor con una muestra de dentina en el centro (Figura 1). La cara pulpar del corte fue lijada para regularizar la superficie usando papel abrasivo (n. 600 Siliconcarbide) bajo agua circulando. Cada disco fue rotulado usando un marcador permanente identificando el número de muestra y las caras pulpar y oclusal de cada una. Cada muestra fue luego etiquetada de acuerdo a la fecha de exodoncia y conservada sumergida en solución de Hank hasta su utilización (Figura 1). Para medir la conductancia hidráulica de los discos de dentina tres equipos fueron montados usando como referencia el modelo original de Pashley. Estas estaban constituidas por las siguientes partes:

- Columna de Agua: Se montó una bureta sostenida por una pinza de bureta sobre un soporte universal. En el soporte universal se montó además una regla milimetrada. La bureta se llenó de agua destilada y el espejo de agua en el interior de ésta se hizo coincidir con el nivel $40 \mathrm{~cm}$ desde la base del soporte universal medido por la regla montada en él.
- Capilar Milimetrado: La bureta se conectó en su extremo mediante tubos de silicona a una pipeta de $0.01 \mathrm{ml}$ en posición horizontal para ser usada como capilar milimetrado. En su interior se ingresó una burbuja de aire de $10 u l$ usando una microjeringa, la cual permitió la medición visual de tasa de difusión, medido en relación al desplazamiento de la burbuja en el interior del capilar (Figura 2).

- Cámara de Difusión: En el otro extremo de la pipeta, usando capilares de silicona, se conectó un portafiltro atornillable de $25 \mathrm{~mm}$ de diámetro (Millipore ${ }^{\circledR}$ Swinnex), el cual se sostuvo en su lugar usando una pinza y otro soporte universal. En el soporte universal se adicionó una regla milimetrada. El portafiltro se posicionó en el nivel $20 \mathrm{~cm}$ desde la base del soporte universal medido por la regla montada en éste.

La máquina completa se llenó con agua, evitando que quedaran burbujas en el interior de los tubos de silicona (Figura 2).

Para asegurar que los tres equipos funcionáran de la misma manera y evitar errores en los resultados producto del uso indistinto de ellas, la muestra número uno se utilizó para controlar su correcto funcionamiento.

Previo a medir por primera vez la tasa de filtración de cada muestra, éstas fueron sometidas a tratamiento de superficie tanto en su cara oclusal como pulpar con el objetivo de eliminar la capa de barro dentinario que obliteraba los túbulos dentinarios y que se produjo durante el corte y la regularización de la superficie de cada muestra. Ambas caras fueron grabadas por 30 segundos con ácido fosfórico en gel al $35 \%$ (Coltène $®$ Etchant Gel S). Luego cada muestra fue lavada con agua destilada por un minuto.

Para medir la tasa de flujo de las muestras de dentina, cada muestra fue instalada al interior de la cámara de difusión, sellando la muestra utilizando o-rings de silicona. A través de la máquina, la muestra fue sometida a una presión de $200 \mathrm{~mm}$ de agua destilada en su lado pulpar, midiendo indirectamente gracias al desplazamiento de la burbuja al interior del capilar, la tasa de difusión desde el lado pulpar al lado oclusal de cada muestra. Usando un cronómetro, se registró el tiempo que demoró en difundir a través de la dentina un volumen de 10ul (Figura 2).

La muestra número uno se usó como control para comprobar el correcto funcionamiento de los tres equipos y que éstos validaran el mismo resultado. Para esto, la muestra número uno fue instalada en los tres equipos y medida su tasa de filtración tres veces en cada una el mismo día. Estos valores fueron tabulados y analizados estadísticamente.

Además, se usó un disco sólido de resina epóxica como control negativo y se dejó la cámara de difusión sin interponer ninguna muestra como control positivo.

Las muestras 2 a la 17 fueron instaladas y medida su tasa de filtración tres veces en los días 14, 21, 28 y 35 postextracción. Así se obtuvo para cada muestra 12 medidas independientes tomadas en cuatro tiempos distintos. Una vez utilizada, cada muestra fue retirada de la cámara de difusión y almacenada debidamente identificada, sumergida en solución de Hank ${ }^{(11)}$ hasta su próximo uso.

En cada medición, las muestras fueron observadas durante el tiempo que fuese necesario para que la burbuja al interior del capilar milimetrado se desplazara indicando un volumen de líquido difundido a través de la muestra de $10 \mathrm{ul}$. El tiempo fue registrado y tabulado en una base de datos (Figura 2).

Una vez finalizada la etapa de mediciones del día 35 postextracción, cada muestra fue fotografiada en forma estandarizada sobre una hoja milimetrada a $30 \mathrm{~cm}$ de distancia con una máquina fotográfica Nikoncoolpix D90 (2009 Nikon®Corporation). Las imágenes digitales de las muestras fueron procesadas usando el programa de Procesamiento y Análisis de imagen ImageJ (@NIH 2010)(12). De esta forma, fue posible calcular el área de dentina de cada muestra. Estos datos fueron tabulados.

Usando la tasa de filtración obtenida en las mediciones de cada muestra y el área de cada muestra, se calculó la conductancia hidráulica de la dentina de cada una de ellas. Los datos fueron tabulados y analizados estadísticamente usando el programa computacional Stata 11 (@2009 StataCorp LP) usando las pruebas estadísticas de ShapiroWilk y Kruskal-Wallis.

El Test ANOVA se utilizó en las situaciones en las que nos interesa analizar una respuesta cuantitativa. El objetivo principal del ANOVA es contrastar si existen diferencias entre las diferentes medias de los niveles de las variables. Cuando el análisis de la varianza no es aplicable debido a incumplimientos de las suposiciones del modelo (distribución normal) utilizamos la prueba de Kruskal-Wallis. 


\section{RESULTADOS}

De los 17 terceros molares utilizados en el estudio se obtuvo una muestra o disco de dentina de aproximadamente $1 \mathrm{~mm}$ identificado con números correlativos.

La tasa de flujo del control negativo fue 0 . La tasa de flujo del control positivo fue $6.338 \mu \mathrm{L} / \mathrm{min}$. La muestra 1 fue utilizada para comprobar el funcionamiento de los 3 equipos. Se sometió a medición de tasa de flujo 3 veces en cada equipo en el mismo día. Los resultados se presentan en la siguiente tabla (Tabla 1). El análisis ANOVA nos muestra que el valor de "p" para la tasa de flujo de la muestra 1 en las 3 equipos es " $p=0.5937$ ". Esto significa que no existe diferencia significativa en las medidas realizadas en los 3 equipos.

Las muestras de la 2 a la 17, fueron medidas 3 veces los días $7,14,21$ y 28 postextracción. Se realizó un análisis descriptivo de las mediciones de conductancia hidráulica (Tabla 2).

En total fueron 168 mediciones de conductancia hidráulica. El promedio de la conductancia hidráulica de las muestras fue de $2.330541 \mu \mathrm{L} / \mathrm{min}$, con una desviación estándar de 2.802809 $\mathrm{L} / \mathrm{min}$ (Gráfico 1). El test de ShapiroWilk indica que la variable conductancia no tiene distribución normal.

Al eliminar los datos extremos, explícitamente los datos de la muestra 16, la prueba Kruskal-Wallis entregó un valor de " $p=0.0704$ ", lo que indica que no hay diferencia significativa en las medias de conductancia hidráulica de las muestras en las 4 semanas (Tabla 3 ).

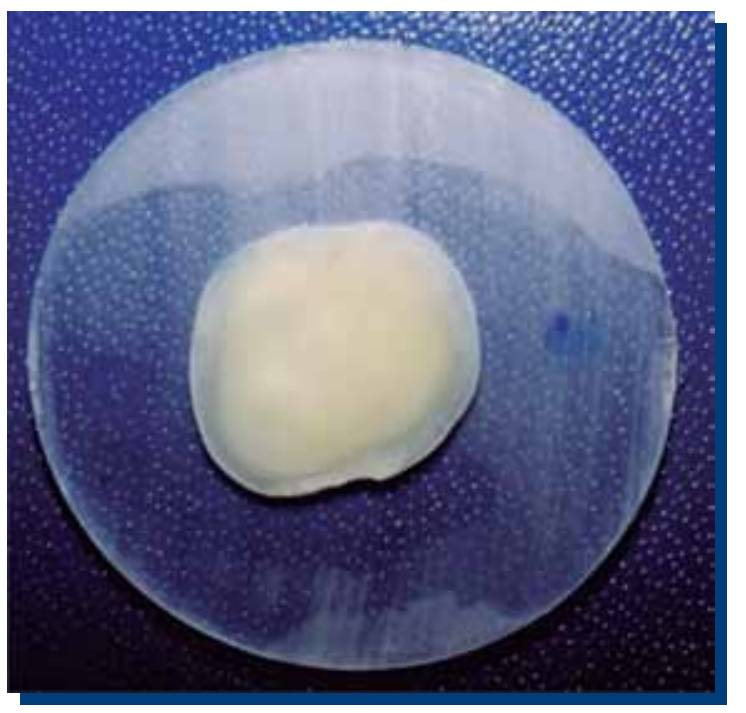

Figura 1. Muestra/Unidad de trabajo: Disco dentinario.

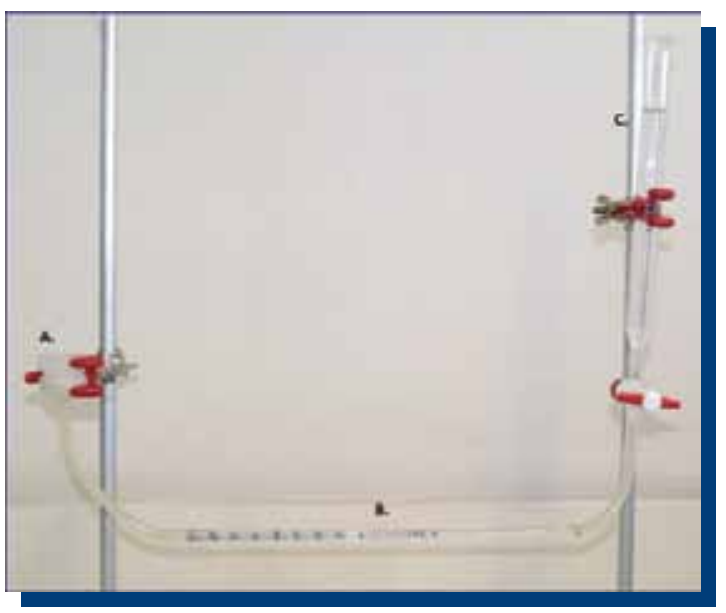

Figura 2. Máquina de difusión. A: Cámara de difusión; B: Capilar milimetrado; C: Columna de agua.
Tabla 1. Tasa de flujo de la muestra 1 en los 3 equipos.

\begin{tabular}{|l|l|l|l|}
\hline Equipo 1 & 0.650759219 & 0.637619554 & 0.640341515 \\
\hline Equipo 2 & 0.654307525 & 0.648648649 & 0.641025641 \\
\hline Equipo 3 & 0.648648649 & 0.644468314 & 0.642398287 \\
\hline
\end{tabular}

Tabla 2. Análisis descriptivo medidas de tasa de flujo de la muestra 1 en los 3 equipos.

\begin{tabular}{|c|c|c|c|c|c|}
\hline Variable & $\mathbf{N}^{\circ}$ & Media & Desv. Est. & Min. & Máx. \\
\hline $\begin{array}{c}\text { Tasa de } \\
\text { Flujo }\end{array}$ & 9 & 0.6453575 & 0.005526 & 0.6376196 & 0.6543075 \\
\hline
\end{tabular}

Tabla 3. Conductancia hidráulica de las muestras en los días 7, 14, 21 y 28 postextracción expresada en $\mu \mathrm{L} / \mathrm{min} . \mathrm{cm}^{2}$ a $20 \mathrm{cmH}^{2} \mathrm{O}$.

\begin{tabular}{|c|c|c|c|c|c|c|}
\hline \multicolumn{7}{|c|}{ Conductancia hidráulica ( $\left.\mu \mathrm{L} / \mathrm{min} . \mathrm{cm}^{2}\right)$} \\
\hline \multirow{2}{*}{$\frac{\text { Muestra }}{2}$} & \multicolumn{3}{|l|}{ Día 7} & \multicolumn{3}{|l|}{ Día 14} \\
\hline & 0.57954216 & 0.57920145 & 0.55914965 & 0.93297507 & 1.00124154 & 0.98325516 \\
\hline 3 & 1.52998776 & 1.51998784 & 1.51750825 & 1.37405105 & 1.262188 & 1.23865853 \\
\hline 4 & 0.90906336 & 0.91130427 & 0.9143094 & 1.16131655 & 1.2268333 & 1.17236501 \\
\hline 5 & 1.09809864 & 1.11794923 & 1.12441671 & 1.02837809 & 0.9767008 & 0.91928663 \\
\hline 6 & 1.43535519 & 2.0006869 & 1.46218426 & 4.03231226 & 3.81594429 & 3.97090649 \\
\hline 9 & 1.3053467 & 1.76497582 & 2.05431612 & 2.30167255 & 2.22889239 & 2.22011722 \\
\hline 10 & 4.48095594 & 4.48095594 & 3.23624595 & 5.27648797 & 5.35408338 & 5.10986203 \\
\hline 11 & 3.23944346 & 2.65270753 & 2.16187391 & 1.69911986 & 1.56370128 & 1.39018897 \\
\hline 12 & 2.29510454 & 2.48083555 & 2.49525901 & 2.54708931 & 2.49525901 & 2.39767905 \\
\hline 13 & 0.71179021 & 0.68019807 & 0.68205147 & 1.50187735 & 1.44134102 & 1.51398926 \\
\hline 14 & 0.63918185 & 0.60938947 & 0.62027813 & 1.34228188 & 1.22620147 & 1.19137445 \\
\hline 15 & 0.45386054 & 0.46670307 & 0.46307873 & 1.24629228 & 1.10583994 & 1.1871268 \\
\hline 16 & 15.6188989 & 16.374652 & 17.2072615 & 1.80967634 & 1.86966561 & 1.77177736 \\
\hline 17 & 0.83718205 & 0.83382436 & 0.81743201 & 1.48323573 & 1.40631482 & 1.36926591 \\
\hline Muestra & \multicolumn{3}{|l|}{ Día 21} & \multicolumn{3}{|l|}{ Día 28} \\
\hline 2 & 0.99316701 & 1.00124154 & 1.00430344 & 1.06051849 & 1.03598494 & 1.03816826 \\
\hline 3 & 1.21124031 & 1.24031008 & 1.22077764 & 1.07293259 & 1.04403205 & 1.05349101 \\
\hline 4 & 1.19898087 & 1.2094409 & 1.23640725 & 1.10244265 & 1.10025526 & 1.11575181 \\
\hline 5 & 0.96219534 & 0.99237361 & 1.01155704 & 1.03071532 & 0.93701392 & 0.96219534 \\
\hline 6 & 3.8535398 & 3.95085141 & 3.89188348 & 2.71621034 & 2.51533305 & 2.66985863 \\
\hline 9 & 2.27382974 & 2.16472082 & 2.2157555 & 1.88283731 & 1.7271356 & 1.71401147 \\
\hline 10 & 5.18260028 & 5.21975154 & 5.09199538 & 5.47485218 & 6.55995802 & 6.27720121 \\
\hline 11 & 1.52696234 & 1.64535024 & 1.69911986 & 1.41670484 & 1.40711956 & 1.36823862 \\
\hline 12 & 3.32701201 & 3.50354734 & 3.54697975 & 2.89013165 & 2.77789352 & 2.64113569 \\
\hline 13 & 0.45594334 & 0.47199162 & 0.46758323 & 0.88345726 & 0.89504014 & 0.88138342 \\
\hline 14 & 0.64408919 & 0.64991053 & 0.64781944 & 1.06983147 & 1.0475665 & 1.07784947 \\
\hline 15 & 1.99278611 & 1.98259283 & 2.01349039 & 1.22270315 & 1.24031008 & 1.2543589 \\
\hline 16 & 7.63329644 & 6.72336706 & 7.41042647 & 14.1003948 & 10.686615 & 13.7193031 \\
\hline 17 & 0.9065416 & 0.9000009 & 0.82445555 & 0.89227557 & 0.90130148 & 0.89676581 \\
\hline
\end{tabular}




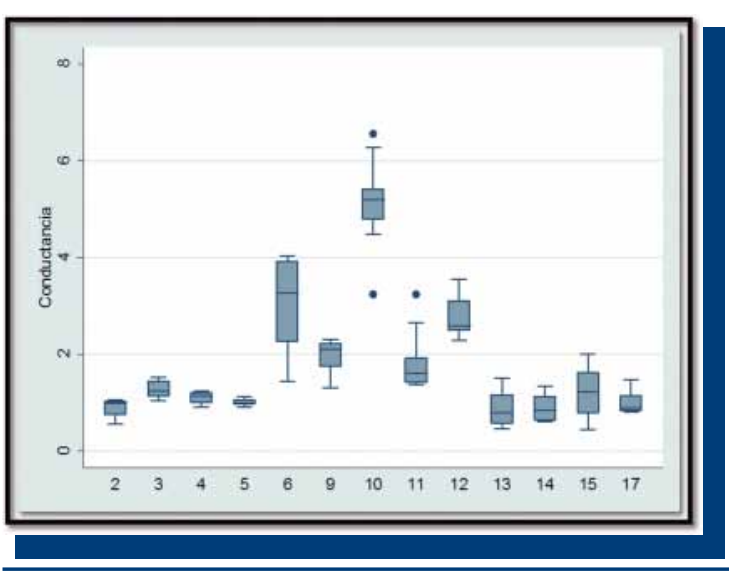

Gráfico 1. Conductancia hidráulica de las muestras, eliminada la muestra 16

\section{DISCUSIÓN}

El modelo experimental utilizado, está basado en los estudios de difusión de Pashley y cols.(13-15). Este modelo ha sido ampliamente usado para estudios de este tipo, pero en ninguna publicación aparecen especificaciones técnicas de cómo debe montarse el equipo para medir la difusión a través de la dentina ${ }^{(6,7,9,16,17)}$.

El equipo utilizado en este trabajo, fue montado en base a las descripciones encontradas en la literatura, pero con materiales fáciles obtener y de bajo costo. La mayor diferencia con el modelo propuesto por Pashley y col. ${ }^{(18)}$, y otros descritos en la literatura, corresponde a la cámara de difusión. En este estudio la cámara de difusión corresponde a un porta filtro, el cual es capaz de sostener muestras y mantener el sello perimetral, pero tiene la limitación que no permite asegurar en su interior muestras mayores a $1.3 \mathrm{~mm}$ de grosor.

El modelo se ve limitado también en cuanto a sensibilidad, ya que el capilar milimetrado fue reemplazado por una pipeta y no permite realizar mediciones de menores a $0.001 \mathrm{ml}$. De necesitar una escala de medida que permita mayor sensibilidad, esta pipeta puede ser fácilmente reemplazada por otro tipo de capilar graduado.

Se utilizó una presión hidrostática de $20 \mathrm{cmH}_{2} \mathrm{O}$, siguiendo el modelo propuesto por Pashley ${ }^{(19)}$.

Al montar 3 equipos para medir la tasa de flujo de agua de las muestras de dentina, fue necesario primero saber si las 3 registraban las mismas mediciones. Las mediciones no presentaron diferencia significativa. Con este resultado, se procedió a utilizar indistintamente cualquiera de ellas para medir la conductancia hidráulica del resto de las muestras.

Tres de las 17 muestras eliminadas y no consideradas para los análisis estadísticos de este estudio. Las muestras 7 y 8 , no presentaron flujo de fluido. Al ser analizadas y observadas con lupa, se evidenció la presencia de esmalte remanente en la superficie oclusal de la muestra. No presentaban dentina expuesta en la superficie, por lo que no permitían evaluar la tasa de flujo a través de ésta. Distinto es lo que ocurrió con la muestra 16 . Ésta, presentó resultados muy dispares y que no seguían ninguna tendencia. Por lo tanto se presumió que el diente no quedó sellado en la resina epóxica, permitiendo el paso de líquido por la interface de la resina y el diente.

La conductancia hidráulica de las muestras no tiene distribución normal, por lo que se realizó la prueba de Kruskall Wallis. Al analizar todas las mediciones, el valor "p" fue igual a 0.2559 . Este resultado significa que las mediciones de conductancia hidráulica obtenidas con este modelo experimental no presentan diferencia estadísticamente significativa entre ellas.

El posible defecto en la muestra 16 , hace reflexionar sobre el método para obtener las muestras de dentina. La resina epóxica no tiene unión química con la superficie del diente, y no es lo suficientemente fluida como para producir una unión micromecánica con la superficie del diente grabado con ácido ortofosfórico al 35\%. Se usó cianoacrilato para intentar lograr una unión micromecánica con el diente, pero se tuvo la certeza que ésta lograra sellar la interface entre el diente y la resina epóxica. Se sugiere estudiar microscópicamente esta interface y modificar si fuese necesario, los procedimientos para obtener las muestras de dientes.

De acuerdo a los resultados obtenidos, el modelo experimental permite estudiar la conductancia hidráulica de la dentina ex vivo. Sin embargo, sería necesario aumentar el " $n$ " de las muestras para poder validar la confiabilidad este modelo experimental, y así poder utilizarlo en estudios de otras variables tales como enfermedades sistémicas, tratamientos de superficie, efectos de instrumental de corte $u$ otros y su influencia en la conductancia hidráulica de la dentina.

\section{CONCLUSIONES}

- Es posible montar una modelo experimental para estudiar la conductancia hidráulica de discos de dentina ex vivo, basándose en el modelo de Pashley y cols., con materiales simples y fáciles de obtener. - No existe diferencia estadísticamente significativa en las mediciones repetidas de la conductancia hidráulica medidas en los 3 equipos.

- No existe diferencia estadísticamente significativa en las mediciones de la conductancia hidráulica de 13 muestras de dentina humana medida en los días 14, 21, 28 y 35 postextracción.

- El modelo experimental pareciera ser confiable con el " $n$ " estudiado, pero se sugiere complementar con un mayor número de muestras poder lograr una mayor confiabilidad.

\section{CONFLICTO DE INTERESES}

Los autores declaran no presentar conflictos de interés de ningún tipo.

\section{REFERENCIAS BIBLIOGRÁFICAS}

1. Reis C, De-Deus G, Marins J, Fidel S, Fidel R, Paciornik S. Mapping large extensions of flat dentin through digital microscopy: Introduction to the method and possible applications. J Adhes Dent, 2012; 14(4): 349-354.

2. Turrioni AP, de Oliveira CF, Basso FG, Moriyama LT, Kurachi C, Hebling $\mathrm{J}$ et al. Correlation between light transmission and permeability of human dentin. Lasers Med Sci, 2012; 27(1): 191-196.

3. Reeder OW, Walton RE, Livingston MJ, Pashley DH. Dentin permeability: Determinants of hydraulic conductance. J Dent Res, 1978; 57(2): 187-193.

4. Pashley DH, Carvalho RM. Dentine permeability and dentine adhesion. J Dent, 1997; 25(5): 355-372.

5. Gillam DG, Mordan NJ, Newman HN. The dentin disc surface: A plausible model for dentin physiology and dentin sensitivity evaluation. Adv Dent Res, 1997; 11(4): 487-501. 6. Lanza CR, de Souza Costa CA, Furlan M, Alecio A, Hebling J. Transdentinal diffusion and cytotoxicity of self-etching adhesive systems. Cell Biol Toxicol, 2009; 25(6): 533-543. 7. Lessa FC, Nogueira I, Huck C, Hebling J, Costa CA. Transdentinal cytotoxic effects of different concentrations of chlorhexidine gel applied on acid-conditioned dentin substrate. J Biomed Mater Res B Appl Biomater, 2010; 92(1): 40-47.

8. Lessa FC, Nogueira I, Vargas Fda S, Spolidorio DM, Hebling J, Garcia-Godoy $\mathrm{F}$ et al. Direct and transdentinal antibacterial activity of chlorhexidine. Am J Dent, 2010; 23(5): 255-259.

9. Soares DG, Ribeiro AP, Sacono NT, Coldebella CR, Hebling J, Costa CA. Transenamel and transdentinal cytotoxicity of carbamide peroxide bleaching gels on odontoblast-like MDPC-23 cells. Int Endod J, 2011; 44(2): 116-125.

10. de Souza BD, Bortoluzzi EA, da Silveira Teixeira C, Felippe WT, Simões CM, Felippe MC. Effect of HBSS storage time on human periodontal ligament fibroblast viability. Dent Traumatol, 2010; 26(6): 481-483.
11. Thomas T, Gopikrishna V, Kandaswamy D. Comparative evaluation of maintenance of cell viability of an experimental transport media "coconut water" with Hank's balanced salt solution and milk, for transportation of an avulsed tooth: An in vitro cell culture study. J Conserv Dent, 2008; 11(1): 22-29.

12. Schneider CA, Rasband WS, Eliceiri KW. NIH Image to ImageJ: 25 years of image analysis. Nat Methods, 2012; 9(7): 671-675 human dentine according to the dentinal region and the composition of the simulated dentinal fluid. J Dent, 2002; 30(2-3): 107-111.

14. Ozok AR, Wu MK, Wesselink PR. The effects of post-extraction time on the hydraulic conductance of human dentine in vitro. Arch Oral Biol, 2002; 47(1): 41-46.

15. Ozok AR, De Gee AJ, Wu MK, Wesselink PR. The influence of resin composite and bonded amalgam restorations on dentine permeability in Class II cavities in vitro. Dent Mater, 2001; 17(6): 477-484.

16. da Costa JB, McPharlin R, Hilton T, Ferracane JI, Wang M. Comparison of two at-home whitening products of similar peroxide concentration and different delivery methods. Oper Dent, 2012; 37(4): 333-339.

17. Lima AF, Lessa FC, Mancini MN, Hebling J, Costa CA, Marchi GM. Transdentinal protective role of sodium ascorbate against the cytopathic effects of $\mathrm{H}_{2} \mathrm{O}_{2}$ released from bleaching agents. Oral Surg Oral Med Oral Pathol Oral Radiol Endod, 2010; 109(4): e70-76.

18. Pashley EL, Tao L, Derkson G, Pashley DH. Dentin permeability and bond strengths after various surface treatments. Dent Mater, 1989; 5(6): 375-378.

19. Ozok AR, Wu MK, ten Cate JM, Wesselink PR. Effect of perfusion with water on demineralization of human dentin in vitro. J Dent Res, 2002; 81(11): 733-737.
13. Ozok AR, Wu MK, Wesselink PR. Comparison of the in vitro permeability of 